\title{
Statistical-Based Approach for Extracting 3D Blood Vessels from TOF-MyRA Data
}

\author{
M. Sabry Hassouna ${ }^{1}$, Aly A. Farag ${ }^{1}$, Stephen Hushek ${ }^{1}$, and Thomas Moriarty ${ }^{2}$ \\ ${ }^{1}$ Computer Vision and Image Processing Laboratory \\ University of Louisville, Louisville, KY 40292 \\ \{msabry, farag, hushek\} @cvip. Louisville.edu \\ http://www.cvip.louisville.edu \\ ${ }^{2}$ Department of Neurological Surgery \\ University of Louisville, KY 40292 \\ tmoriarty@niky.com
}

\begin{abstract}
In this paper we present an automatic statistical intensity basedapproach for extracting the 3D cerebrovascular system from time-of-flight (TOF) magnetic resonance angiography (MRA) data. The voxels of the dataset are classified as either background tissues, which are modeled by a finite mixture of one Rayleigh and two normal distributions, or blood vessels, which are modeled by one normal distribution. We show that the proposed models fit the clinical data properly and result in fewer misclassified vessel voxels. We estimated the parameters of each distribution using the expectation maximization (EM) algorithm. Since the convergence of the EM is sensitive to the initial estimate of the parameters, a novel method for parameter initialization, based on histogram analysis, is provided. A new geometrical phantom motivated by a statistical analysis was designed to validate the accuracy of our method. The algorithm was also tested on 20 in-vivo datasets. The results showed that the proposed approach provides accurate segmentation, especially those blood vessels of small sizes.
\end{abstract}

\section{Introduction}

Large numbers of people suffer a major cerebrovascular event, usually a stroke, each year. Serious types of vascular diseases such as carotid stenosis, aneurysms, and arterio-venous malformations (AVM) may lead to brain stroke unless they are detected at early stages. MRA is a non-invasive MRI-based flow imaging technique. Its wide variety of acquisition sequences and techniques, beside its ability to provide detailed images of blood vessels, enabled its use in the diagnosis and surgical planning of the aforementioned diseases. There are three techniques commonly used in performing MRA; TOF angiography, phase contrast angiography (PCA), and contrast enhanced MRA (CE-MRA) [1]. Both TOF and PCA are non-invasive techniques, while CEMRA requires the injection of a contrast agent, commonly gadolinium. PCA provides good background suppression and can quantify the flow velocity vectors for each voxel. The TOF technique is not as quantitative but it is widely used clinically because it is fast and provides high contrast images, which is the main motivation be 
hind our work. Different approaches have been applied to the problem of vascular segmentation in both 2D and 3D. Most of the 2D approaches are not applicable to 3D images. 3D techniques can be classified under two main categories; topological and statistical techniques. In early topological techniques vessels were detected by cylinder matching [2], [3]. A more generalized technique approximating the vessel cross section by a polygon has been developed in [4]. The Vessel centerlines can be detected using a multi-scale 3D filters [5], and has been modeled by a second order Bspline, and then extracted using iterative tracking technique [6]. A geodesic active contour and level set method has been proposed to segment MRA speed images [7], [8]. Adaptive statistical segmentation method for extracting TOF-MRA is presented in [9]. Two different statistical models for segmenting PCA are suggested in [10], [11]. Both speed and phase information provided by PCA are fused together to extract vessels [12]. In this paper, we present a new TOF intensity-based statistical model that will fit the clinical data properly and provides more accurate segmentation, especially for small size blood vessels. The parameters of the proposed model are estimated using the EM algorithm. An automatic method for selecting the initial estimate of the model parameters necessary by the EM is provided to ensure its convergence. A geometrical phantom motivated by a statistical analysis was designed to validate the results.

\section{Statistical Segmentation}

To the best of our knowledge, there is only one related work [9] that segmented blood vessels from TOF datasets using a statistical approach. In that study, the TOF data histogram was divided into three regions, based on voxel intensity. The lowest intensity region corresponds to cerebrospinal fluid (CSF), bone and the background air. The middle intensity region corresponds to brain tissues, including both the grey and white matter, and parts of the eyes. The third high intensity region corresponds to subcutaneous fat, and arteries. A normal distribution is used to model each of the low and middle intensity regions, while a uniform distribution is used to model the vessel class as shown in Fig. 1(a). We have tested various probability density models for the low intensity range of the TOF histogram, and found that the Rayleigh distribution provides an accurate fit when compared with the normal distribution proposed by [9], as shown in Fig. 1(b). Theoretically, the vessel intensities are uniformly distributed over the high intensity range motivated by the physical model provided by [10], but since the starting point of that range is unknown it is extended over the whole intensity range [9]-[12]. When we first modeled the vessel class by a uniform distribution, we found that the decision level exceeds the expected value found from manual segmentation by 5 to 15 pixels, which lead to the exclusion of small branches. Thus, the assumption that vessels exist in the low intensity range biases the fitting process, which lead to inaccurate decision level value as well. Using a normal distribution as a model to the vessel class reduced the absolute error between the observed data histogram and the proposed model and improved the decision level. These improvements resulted because the class peak exists in the upper part of the high intensity region, thus ensuring very low contribution by its long left tail to the low and middle intensity regions. Modeling the middle intensity region by one normal distribution, leads to an accurate fitting at both ends of the histogram but not at the middle as marked by the 
circle in Fig. 1(b). To correct this problem, an additional normal distribution is used. Hence, the middle intensity region is modeled by a mixture of two normal distributions. Since we are interested only in segmenting blood vessels we assume that the TOF dataset consists of only two major classes, background and vessels, where background includes both the low and middle intensity regions. Thus, a mixture of three distributions (one Rayleigh and two normal) models the background class, while a normal distribution models the vessel class, as shown in Fig. 1(c).

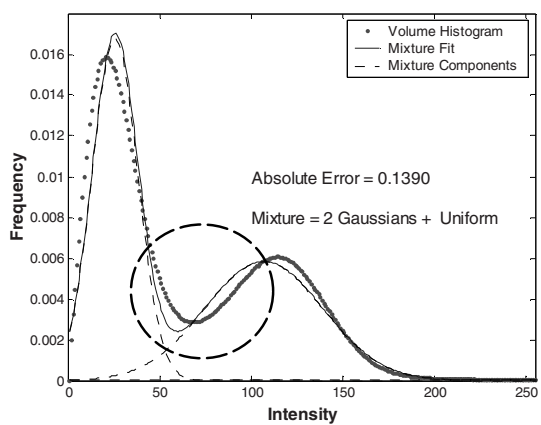

(a)

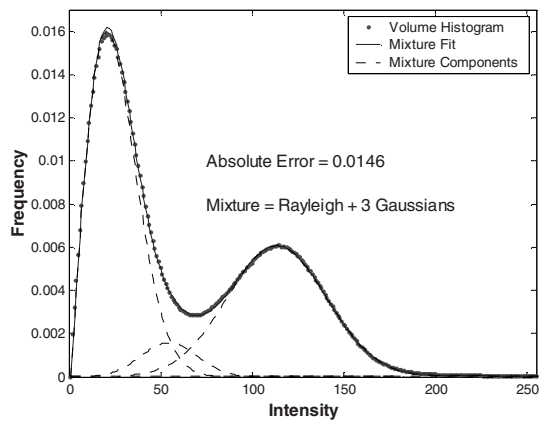

(c)

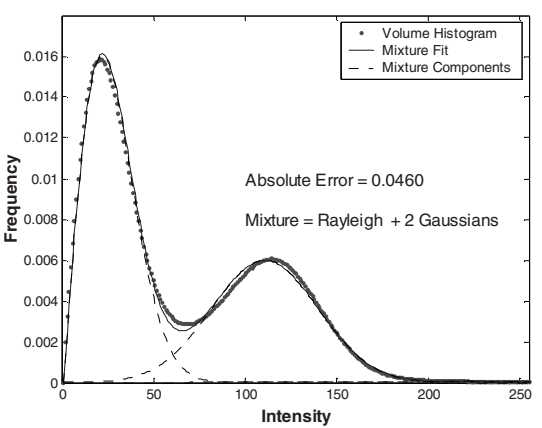

(b)

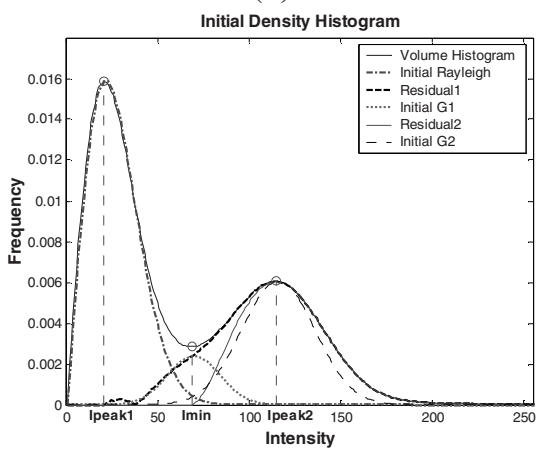

(d)

Fig. 1. (a) Model by [9] (b) Inaccuracy of Rayleigh and two normal. (c) The proposed model (accurate fitting) (d) Initial histogram of each distribution

The total probability density function of the mixture is given by Eq. (1)

$$
f(x)=\underbrace{w_{R} f_{R}(x)+w_{G 1} f_{G 1}(x)+w_{G 2} f_{G 2}(x)}_{\text {Background Signal }}+\underbrace{w_{G 3} f_{G 3}(x)}_{\text {Vessel Signal }}
$$

The functions $f_{R}(x), f_{G 1}(x), f_{G 2}(x)$, and $f_{G 3}(x)$ are the Rayleigh and normal density functions, respectively. The quantities $w_{R}, w_{G 1}, w_{G 2}$, and $w_{G 3}$ are the class proportions which sum is unity. The maximum a posteriori (MAP) classification of a voxel $x_{i}$ to the vessel class can be obtained by the following equation: 


$$
\text { IF } w_{G 3} f_{G 3}\left(x_{i}\right)>w_{R} f_{R}\left(x_{i}\right)+\sum_{l=1}^{2} w_{G l} f_{G l}\left(x_{i}\right), \text { THEN } x_{i} \rightarrow \text { vessel }
$$

\section{Parameter Estimation}

There are eleven parameters in Eq. (2) that needs to be estimated before applying the segmentation; $w_{R}, \beta, w_{G l}, \mu_{G l}$, and $\sigma_{G l}^{2} \forall l \in[1,3]$, where $\beta$ is the Rayleigh mode. We estimated those parameters using the EM algorithm [13]-[14]. In the literature there is a closed form for the update equations of the mean and variance of the normal distribution and the proportion of any distribution [14]. The update equation for the mode of the Rayleigh distribution at iteration $k+1$ can be derived by maximizing the conditional expectation with respect to it; that is,

$$
\left(\beta^{2}\right)^{k+1}=\sum_{i=1}^{N} x_{i}^{2} f\left(R \mid x_{i}, \Theta^{k}\right) / 2 \sum_{i=1}^{N} f\left(R \mid x_{i}, \Theta^{k}\right), f\left(R \mid x_{i}, \Theta^{k}\right)=\frac{w_{R}^{k} f_{R}\left(x_{i} \mid \Theta^{k}\right)}{f\left(x_{i}\right)}
$$

where $\Theta^{k}$ is the estimate of the Rayleigh parameters at iteration $k . N$ is the total number of voxels in the data volume. The EM algorithm is an iterative technique that starts with an initial estimate of the model parameters. During its operation it searches for those parameters that maximize the conditional expectation, thus it may converge to local maxima if the initial values of the parameters are not selected properly [14]. Thus, we developed an automatic method for choosing them. The initial values of the parameters are set according to Table 1 . Let $h(x)$ be the normalized observed histogram and $h_{R}^{\text {init }}(x)$ and $h_{G 1}^{\text {init }}(x)$ be the initial histograms of the Rayleigh and normal distributions, respectively, as defined by Eq. (4). Let $I_{\text {peak } 1}$ and $I_{\text {peak } 2}$ be the intensities at which $h(x)$ achieves its two global peaks, and $I_{\min }$ be the intensity at which $h(x)$ achieves its minimum value between those peaks as shown in Fig. 1(d).

$I_{\text {peak } 2}$ and $I_{\min }$ can be achieved by smoothing $h(x)$ couple of times.

$$
\begin{gathered}
h_{R}^{\text {init }}(x)=C_{R} \cdot f_{R}\left(x \mid \beta^{2 \text { init }}\right) \text { where, } C_{R}=\frac{h\left(I_{\text {peak } 1}\right)}{f_{R}\left(I_{\text {peak } 1} \mid \beta^{2 \text { init }}\right)} \\
h_{G 1}^{\text {init }}(x)=C_{1} \cdot f_{G 1}\left(x \mid \mu_{G 1}^{\text {init }}, \sigma_{G 1}^{2 \text { init }}\right) \text { where, } C_{1}=\frac{h_{r e s 1}\left(\mu_{G 1}^{\text {init }}\right)}{f_{G 1}\left(\mu_{G 1}^{\text {init }} \mid \mu_{G 1}^{\text {init }}, \sigma_{G 1}^{2 \text { init }}\right)}
\end{gathered}
$$

$C_{R}$ and $C_{1}$ of Eq. (4) ensure that the peaks of the initial histograms have the same height as $h(x)$. The residual histograms are calculated according to Eq. (5).

$$
\begin{aligned}
& h_{\text {res } 1}^{\text {init }}(x)=\left|h(x)-h_{R}^{\text {init }}(x)\right| u\left(x-I_{\text {peak } 1}\right) \\
& h_{\text {res } 2}^{\text {init }}(x)=\left|h_{r e s 1}(x)-h_{G 1}^{\text {init }}(x)\right| u\left(x-\mu_{G 1}^{\text {init }}\right)
\end{aligned}
$$


The unit step ensures that $\mu_{G 1}^{\text {init }}$ and $\mu_{G 2}^{\text {init }}$ have values greater than $I_{\text {peak } 1}$ and $\mu_{G 1}^{\text {init }}$, respectively. Once the parameters are estimated, Eq. (2) is used in classification.

Table 1. The initial parameter values needed by the EM algorithm

\begin{tabular}{|c|c|}
\hline Parameter & Value \\
\hline$\mu_{G 1}^{\text {init }}$ & $I_{\min }$ \\
\hline$\mu_{G 2}^{i n i t}$ & $I_{\text {peak } 2}$ \\
\hline$\mu_{G 3}^{i n i t}$ & $\begin{array}{l}\text { Calculated using MLE from the last } 3 \% \text { of the high intensity data } \\
\text { of the observed histogram }\end{array}$ \\
\hline$\beta^{\text {init }}$ & $I_{\text {peak } 1}$, the value at which Rayleigh achieves maximum value. \\
\hline$\sigma_{G 1}^{2 \text { init }}$ & $\begin{array}{l}\text { Calculated using MLE from the samples in the region } \\
{\left[\mu_{G 1}^{\text {init }}-\Delta, \mu_{G 1}^{\text {init }}+\Delta\right] \text { of } h_{\text {res }}^{\text {init }}(x) \text {, where } \Delta=\left(\mu_{G 1}^{\text {init }}-I_{\text {peak } 1}\right) / 2}\end{array}$ \\
\hline$\sigma_{G 2}^{2 \text { init }}$ & $\begin{array}{l}\text { Calculated using MLE from the samples in the region } \\
{\left[\mu_{G 2}^{\text {init }}-\Delta, \mu_{G 2}^{\text {init }}+\Delta\right] \text { of } h_{\text {res } 2}^{\text {init }}(x) \text {, where } \Delta=\left(\mu_{G 2}^{\text {init }}-\mu_{G 1}^{\text {init }}\right)}\end{array}$ \\
\hline$\sigma_{G 3}^{2 \text { init }}$ & $\begin{array}{l}\text { Calculated using MLE from the last } 3 \% \text { of the high intensity data } \\
\text { of the observed histogram }\end{array}$ \\
\hline$w_{G 3}^{\text {init }}$ & $\begin{array}{l}\text { Set to } 3 \% \text { because the proportion of the vessels in the volume } \\
\text { ranges from } 1 \% \text { to } 5 \% \text {. }\end{array}$ \\
\hline$w_{R}^{\text {init }}$ & The area of $h(x)$ covered by $h_{R}^{\text {init }}(x)$, \\
\hline$w_{G 2}^{\text {init }}$ & The area of $h(x)$ covered by $h_{G 2}^{\text {init }}(x)$, \\
\hline$w_{G 1}^{\text {init }}$ & $1-w_{R}^{\text {init }}-w_{G 2}^{\text {init }}-w_{G 3}^{\text {init }}$ \\
\hline
\end{tabular}

\section{Evaluation of Segmentation}

In MRA segmentation, it is quite difficult to devise validation criteria because the actual shape of the 3D vessels is unknown and even expert radiologists differ in the evaluation. Thus, to validate the accuracy of our method, we created a geometrical phantom of blood vessels with varying cross sections such that its histogram and mixture component proportions mimic the real TOF data. The vessel phantom is drawn by hand in a $512 \times 512$ image with a constant inten-

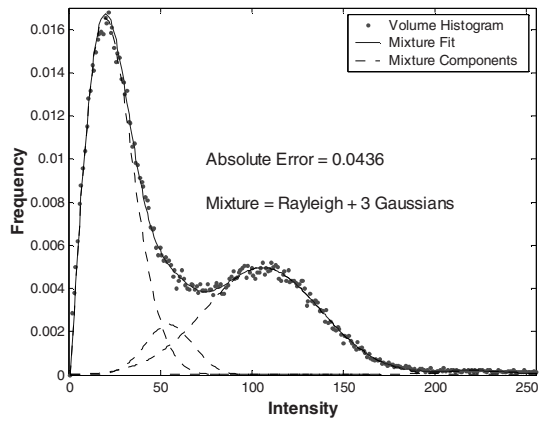

Fig. 2. Accurate fitting of the phantom histogram using the proposed model. 
sity $\mu_{G 3}$ such that its proportion is $1 \%$, as shown in Fig. 3(a). The background signal is generated as follows: three types of voxels are randomly generated over the image of a constant intensity, $\mu_{R}, \mu_{G 1}$, and $\mu_{G 2}$ of proportions $w_{R}, w_{G 1}$, and $w_{G 2}$ respectively. Thus, the histogram of the ground truth phantom consists of impulses at intensities $\mu_{R}, \mu_{G 1}, \mu_{G 2}$, and $\mu_{G 3}$. We then reshaped it to form the TOF signature histogram as follows: we added three independent normal noise components of zero mean and variance $\sigma_{G l}^{2}$ with proportions $w_{G l}$ to the voxel intensities marked by $\mu_{G l}$, which will imply $G_{l} \sim\left(\mu_{G l}, \sigma_{G l}^{2}\right) \forall l \in[1,3]$. We also replaced each voxel marked with $\mu_{R}$ by a Rayleigh noise of mode $\beta=I_{\text {peak } 1}$ to form a Rayleigh distribution. The parameters used in the phantom design are the average values of those extracted from the EM approach as applied to real data of several patients. This analysis is also applicable to 3D phantoms. The phantom histogram is shown in Fig. 2. Fig. 3(a) is a zoomed version of the phantom. Fig. 3(b) shows the phantom with added noise. Fig. 3(c, d) shows the phantom segmentation by our method without and with a connectivity filter[11], respectively. The absolute error between the ground truth and the segmented phantom of Fig. 3(a, d), respectively, was $10 \%$. The reason behind this large error is that during phantom fabrication we distributed the voxels of the vessels normally over the whole intensity range while practically they are distributed only over the high intensity range. Thus, when we distributed them uniformly over the upper half of the intensity range but still modeled by a normal distribution, the error was reduced to $3 \%$, as shown in Fig. 3(e, f).

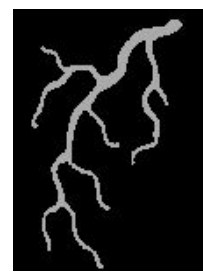

(a)

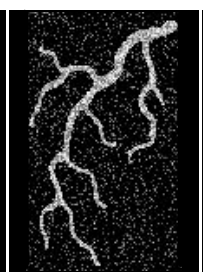

(b)

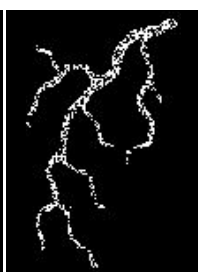

(c)

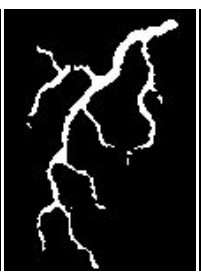

(d)

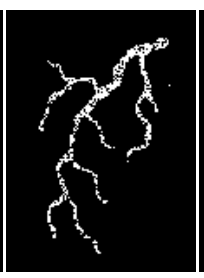

(e)

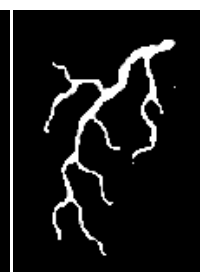

(f)

Fig. 3. (a) Zoom in of the ground truth phantom (b) Noisy phantom. (c, d) Segmentation without and with connectivity filter, respectively, assuming that the voxels of the vessels are distributed normally over the whole intensity range. (e, f) same as (c, d) except that voxels are distributed uniformly over the upper half of the intensity range.

\section{Results}

Dataset were collected using a Picker $1.5 \mathrm{~T}$ Edge MRI scanner. It consists of $512 \times 512 \times 93$ axial slices with slice thickness $1 \mathrm{~mm}, \mathrm{TR}=27 \mathrm{~ms}$, TE $=6 \mathrm{~ms}$. The average processing time taken by our method is approximately 1 minute on a single $400 \mathrm{MHz}$ processor, Onyx2 SGI supercomputer. The proposed segmentation approach is tested on an in-vivo 20 datasets, where three of them are shown in Fig. 4. 
The segmentation results with and without the connectivity filter are shown in Fig. $4(b, c)$, respectively. To show the accuracy of the results, a comparison is done with the maximum intensity projection (MIP) images, as shown in Fig. 4(a). TOF is sensitive to short $\mathrm{T}_{1}$ tissues such as subcutaneous fat, which appears in the segmented volume obscuring vessels as shown in the first row of Fig. 4(b). Therefore, to eliminate the fat, we select one or more (if the tree is not connected) seed points inside the vascular tree, then apply the connectivity filter [11], which is based on region growing algorithm in $3 \mathrm{D}$. The results have also been validated by our neurosurgeon and radiologist. They agreed that the proposed method was successful in showing most of the vessels details, especially small ones.
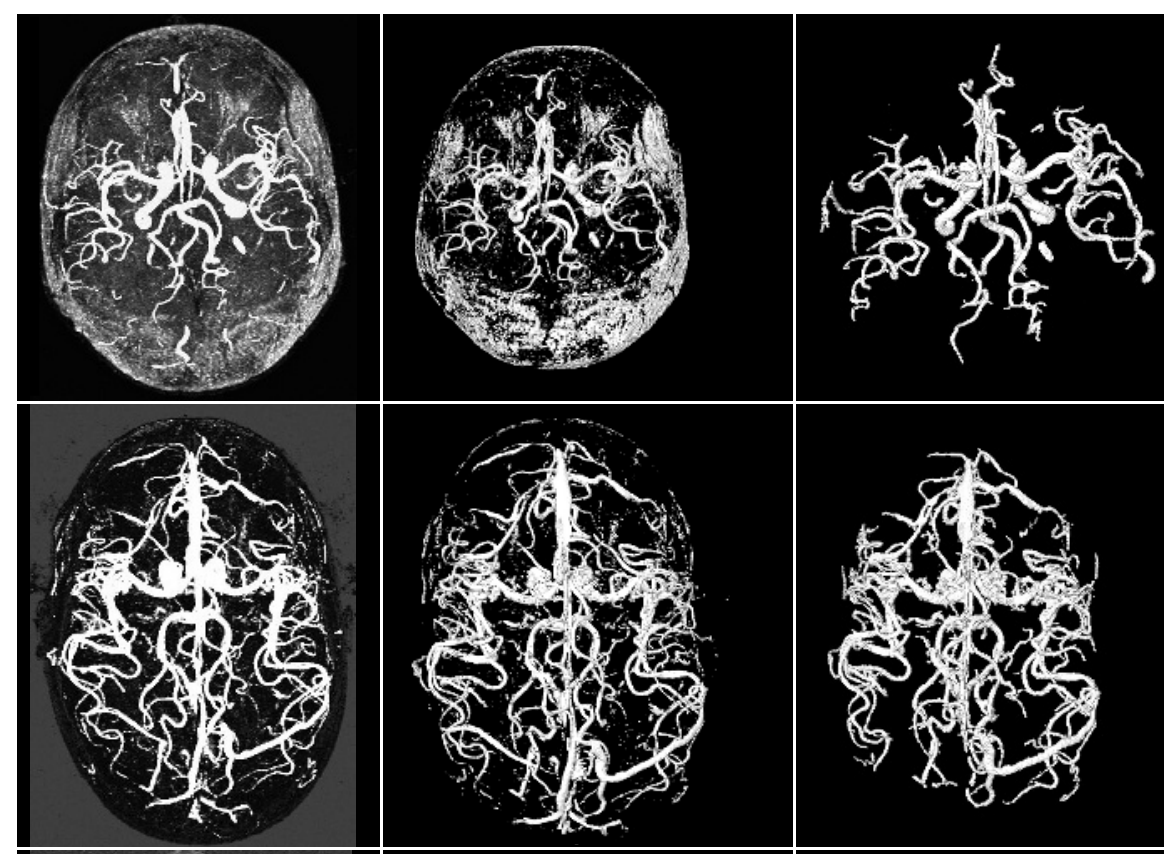

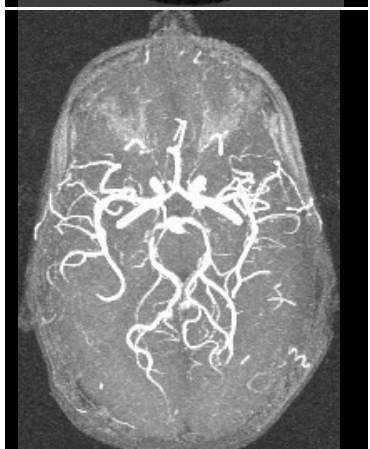

(a)

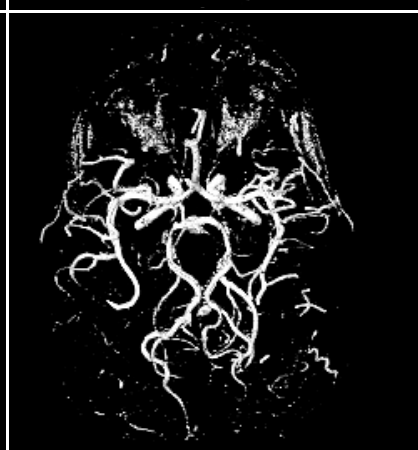

(b)

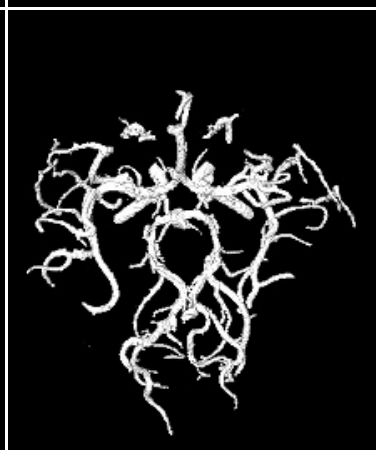

(c)

Fig. 4. Each row represents a patient (a) MIP image (b) Segmentation (c) Segmentation + connectivity filter 


\section{Conclusion and Future Work}

In this paper we suggested a new statistical intensity-based technique for segmenting blood vessels from TOF-MRA data. The proposed model accurately fits the observed data histogram and gives high quality of segmentation validated by a geometrical phantom; analysis of is also applicable to 3D images. Although TOF-2D acquisition has good suppression of background tissues (lower middle peak) more than 3D acquisition, our segmentation technique is still applicable to both of them. We also presented an automatic method based on histogram analysis to choose the initial values of the parameters necessary by the EM to ensure its convergence. Currently we are integrating a Markov Random Field (MRF) model to the segmentation process to take into account the contextual information of neighboring pixels.

Acknowledgments. The Whitaker Foundation Research Grant No. 98-009 has funded this project.

\section{References}

1. Potchen, J. E. et al. Magnetic Resonance Angiography: Concepts and Applications. St. Louis, MO; Mosby, 1993.

2. P. Reuzé, et al., "A 3-D moment based approach for blood vessel detection and quantification in MRA," Technol. Health Care 1, 1993, 181-188.

3. M. Hernandez-Hoyos et al., "Inertia based vessel axis extraction and stenosis quantification in 3D MRA images," CARS, 1999, 180-193.

4. B. Verdonck et al., "A New System for Blood Vessel Segmentation and Visualization in 3D MR and Spiral CT Angiography,". CARS, 1995, 177-182.

5. Y. Sato et al., "Three-dimensional multi-scale line filter for segmentation and visualization of curvilinear structures in medical images,". Medical Image Analysis, 2(2), 1998, 143-168.

6. N. Flasque et al., "Acquisition, segmentation and tracking of the cerebral vascular tree on 3D magnetic resonance angiography images," Med. Image Anal., Vo. 5, No. 3, 2001, 173-183.

7. L.M. Lorigo et al., "Co-dimension 2 geodesic active Contours for MRA segmentation,". In: Kuba, A. (Ed.), Proc. Int. Conf. on Information Processing in Med. Imaging, 1999, 126-139.

8. C. F. Westin, and L. M. Lorigo et al., "Segmentation by adaptive geodesic active contours,”. In: Delp, S.L. (Ed.), Proc. MICCAI, 2000, 266-275.

9. D. Wilson, and J. Noble, "An adaptive segmentation algorithm for time-of-flight MRA data,”. IEEE Trans. Med. Imaging 18 (10), 1999, 938-945.

10. Albert C. S. Chung, and J .Noble, "Statistical 3D Vessel Segmentation Using a Rician Distribution,". MICCAI 1999, 82-89.

11. Mohamed Sabry, A. A. Farag, S. Hushek, and T. Moriarty, "Statistical Cerebrovascular Segmentation for Phase-Contrast MRA Data," Annual Conference on Biomedical Engineering, 2002, pp. 32-37, Egypt.

12. Albert C. S. Chung et al., "Fusing speed and phase information for vascular segmentation of phase contrast MR angiograms," Medical Image Analysis, 2002, Vol. 6, No. 2, 109128.

13. A. P. Dempster, N. M. Laird, and D. B. Rubin, "Maximum likelihood from incomplete data via the EM algorithm," J. R. Statist. Soc. B, vol. B39, no. 1, 1977, pp. 1-38.

14. Geoffrey J. MacLachlan, and Thriyambakam Krishnan, The EM Algorithm and Extensions, Wiley Series in Probability and Statistics, John Wiley and Sons, 1997. 